\title{
TITLE:
}

\section{Ambiguity of black hole entropy in loop quantum gravity}

AUTHOR(S):

Tamaki, T; Nomura, $\mathrm{H}$

\section{CITATION:}

Tamaki, T ... [et al]. Ambiguity of black hole entropy in loop quantum gravity. PHYSICAL REVIEW D 2005, 72(10): 107501.

ISSUE DATE:

2005-11

URL:

http://hdl.handle.net/2433/49872

RIGHT:

Copyright 2005 American Physical Society 
PHYSICAL REVIEW D 72, 107501 (2005)

\title{
Ambiguity of black hole entropy in loop quantum gravity
}

\author{
Takashi Tamaki ${ }^{1, *}$ and Hidefumi Nomura ${ }^{2, \dagger}$ \\ ${ }^{1}$ Department of Physics, Kyoto University, 606-8501, Japan \\ ${ }^{2}$ Department of Physics, Waseda University, 3-4-1 Okubo, Shinjuku, Tokyo 169-8555, Japan
}

(Received 25 August 2005; published 16 November 2005)

\begin{abstract}
We reexmine some proposals of black hole entropy in loop quantum gravity (LQG) and consider a new possible choice of the Immirzi parameter which has not been pointed out so far. We also discuss the fact that a new idea is inevitable if we regard the relation between the area spectrum in LQG and the relation between the area spectrum in the quasinormal mode analysis seriously.
\end{abstract}

DOI: 10.1103/PhysRevD.72.107501

PACS numbers: 04.70.Bw, 04.30.Db, 04.70.Dy

\section{INTRODUCTION}

Loop quantum gravity (LQG) has attracted much attention because of its background independent formulation, account for microscopic origin of black hole entropy [1], singularity avoidance in the universe [2], and black holes [3]. The spin network has played a key role in the development of this theory [4]. Basic ingredients of the spin network are edges and vertices. In Fig. 1, edges are expressed by lines labeled by $j=0,1 / 2,1,3 / 2, \ldots$ reflecting the $\mathrm{SU}(2)$ nature of the gauge group. A vertex is an intersection of edges. In this figure, we write only vertices where three edges merge (we call them trivalent vertices). Even if there is a vertex where more than three edges merge, we can decompose it to the sum of edges and trivalent vertices. For this reason, we consider only trivalent vertices below. For three edges having spin $j_{1}, j_{2}$, and $j_{3}$ that merges at an arbitrary vertex, we have

$$
j_{1}+j_{2}+j_{3} \in N
$$

$$
j_{i} \leq j_{j}+j_{k}, \quad(i, j, k \text { different from each other. })
$$

to guarantee the gauge invariance of the spin network. This is also displayed in Fig. 1.

Using this formalism, general expressions for the spectrum of the area and the volume operators can be derived $[5,6]$. For example, the area spectrum $A$ is

$$
A=4 \pi \gamma \sum \sqrt{2 j_{i}^{u}\left(j_{i}^{u}+1\right)+2 j_{i}^{d}\left(j_{i}^{d}+1\right)-j_{i}^{t}\left(j_{i}^{t}+1\right)},
$$

where $\gamma$ is the Immirzi parameter related to an ambiguity in the choice of canonically conjugate variables [7]. The sum is added up all intersections between a surface and edges as shown in Fig. 1. Here, the indices $u, d$, and $t$ means edges above, below, and tangential to the surface, respectively, (We can determine which side is above or below arbitrarily). If there is no edges which are tangential to the surface, we have $j_{i}^{u}=j_{i}^{d}:=j_{i}$ and $j_{i}^{t}=0$. In this

\footnotetext{
*Electronic address: tamaki@tap.scphys.kyoto-u.ac.jp
}

†Electronic address: nomura@gravity.phys.waseda.ac.jp case, we have the simplified formula as

$$
A=8 \pi \gamma \sum \sqrt{j_{i}\left(j_{i}+1\right)} .
$$

As we will mention below, this is the case for the horizon area spectrum.

The number of states that determines the black hole entropy is basically calculated from (1.3) which was first estimated as [1]

$$
S=\frac{A \ln \left(2 j_{\min }+1\right)}{8 \pi \gamma \sqrt{j_{\min }\left(j_{\min }+1\right)}}
$$

where $A$ and $j_{\min }$ are the horizon area and the lowest nontrivial representation usually taken to be $1 / 2$ because of SU(2), respectively. In this case, the Immirzi parameter is determined as $\gamma=\ln 2 /(\pi \sqrt{3})$ to produce the Bekenstein-Hawking entropy formula $S=A / 4$.

However, the formula (1.4) was corrected as $[8,9]$

$$
S=\frac{\gamma_{M} A}{4 \gamma}
$$

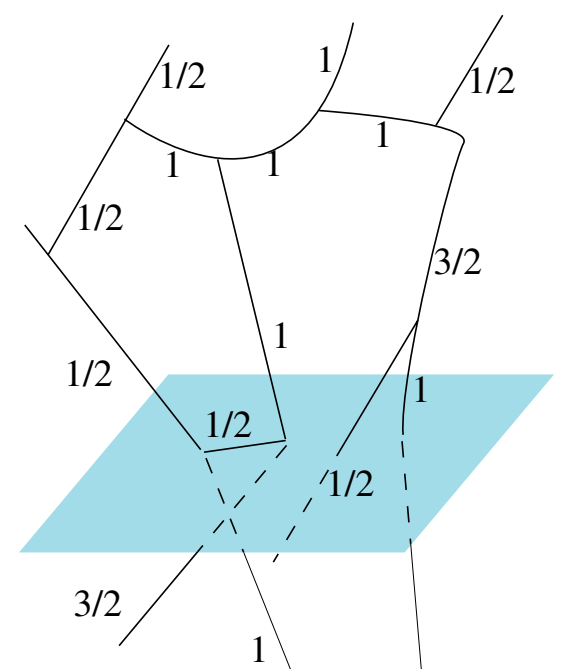

FIG. 1 (color online). Spin network and a surface. 
where $\gamma_{M}$ is the solution of

$$
1=\sum_{j=Z / 2}^{\infty} 2 \exp \left(-2 \pi \gamma_{M} \sqrt{j(j+1)}\right)
$$

where $j$ takes all the positive half-integer. In this case, $\gamma_{M}$ is numerically obtained as $\gamma_{M}=0.23753 \cdots$. This means that it took four years for the original error to be corrected, which suggests that independent reexamining is important. Interestingly, other possibilities have also been argued. One is to determine $\gamma_{M}$ as the solution of [10-12]

$$
1=\sum_{j=Z / 2}^{\infty}(2 j+1) \exp \left(-2 \pi \gamma_{M} \sqrt{j(j+1)}\right)
$$

In this case, $\gamma_{M}=0.27398 \cdots$. The other is to recover (1.4) by imposing the condition that the area is determined only by $j=j_{\min }[13]$.

These provide us with the following question: that is, which is the best choice for the Immirzi parameter? Therefore, we reanalyze these possibilities. This is important because of the following reasons.

(i) In string theory, number counting for microscopic states of black holes has been considered, and it has reproduced the Bekenstein-Hawking formula $S=A / 4$ [14]. In the future, it is desirable for us to have a connection with the number counting in string theory. Although there is no relation between LQG and string theory at present, this link may shed new light on developments to come in theoretical physics. Probably, we will need to proceed with many steps toward this purpose.

However, there is a subject which can be attacked soon. This is (ii) the possible relation to the quasinormal mode which has been argued as another consistency check of the Immirzi parameter in the area spectrum [15]. Using (1.4), an encounter between LQG and the quasinormal mode was considered first in Ref. [16]. This means that if we have $j_{\text {min }}=1$, we can determine $\gamma$ as $\ln 3 /(2 \pi \sqrt{2})$ which gives $A=4 \ln 3$ from (1.3). This coincides with the area spectrum determined by the quasinormal mode using Bohr's correspondence [17]. Moreover, the quasinormal mode analysis that originally performed in Schwarzschild black hole [18-20] has been extended to single-horizon black holes [21-25]. These results suggest that there is a relation between these spectra. However, if we adopt (1.6) or (1.7), we cannot obtain such a consistency. Thus, we also want to know which is the best choice for the Immirzi parameter in this view point.

This paper is organized as follows. In Sec. II, we summarize the framework [1] (which we call the ABCK framework) that is necessary in number counting. In Sec. III, we argue various possibilities that determine the number of state. In Sec. IV, we summarize our results and discuss their meaning.

\section{SUMMARY OF THE ABCK FRAMEWORK}

Here, we briefly introduce the framework in Ref. [1] and summarize the conditions necessary for number counting. First, we introduce the isolated horizon (IH) where we can reduce the $\mathrm{SU}(2)$ connection to the $\mathrm{U}(1)$ connection. This plays the important role of determining the conditions (ii) and (iii) below. For details, see [26]. Moreover, the merit of the $\mathrm{IH}$ is that we can treat the event horizon and the cosmological horizon, where we can define the Hawking temperature in an unified way.

Next, we imagine that spin network pierces the IH. By eliminating the edge tangential to the isolated horizon, we can decompose the Hilbert space as the tensor product of that in the IH $H_{I H}$ and that in the bulk $H_{\Sigma}$, i.e., $H_{I H} \otimes H_{\Sigma}$. If we specify the points that are intersections of edges having $\operatorname{spin}\left(j_{1}, j_{2}, \cdots, j_{n}\right)$ and the $\mathrm{IH}$, we can write $H_{\Sigma}$ as the orthogonal sum

$$
H_{\Sigma}=\bigoplus_{j_{i}, m_{i}} H_{\Sigma}^{j_{i}, m_{i}}
$$

where $m_{i}$ takes the value $-j_{i},-j_{i}+1, \cdots, j_{i}$. This is related to the flux operator eigenvalue $e_{s^{\prime}}^{m_{i}}$ that is normal to the $\mathrm{IH}\left(s^{\prime}\right.$ is the part of the $\mathrm{IH}$ that has only one intersection between the edge with spin $j_{i}$.)

$$
e_{s^{\prime}}^{m_{i}}=8 \pi \gamma m_{i}
$$

Since we eliminate the edge tangential to the $\mathrm{IH}$, we have $m_{i} \neq 0$. That is also the reason why the area spectrum is simplified as (1.3). The horizon Hilbert space can be written as the orthogonal sum similar to (2.1) by eigenstates $\Psi_{b}$ of the holonomy operator $\hat{h}_{i}$, i.e.,

$$
\hat{h}_{i} \Psi_{b}=e^{\left[\left(2 \pi i b_{i}\right) / k\right]} \Psi_{b}
$$

Next, we consider the constraints in the bulk and at the IH, respectively. In the bulk, the Gauss constraint is already satisfied and the diffeomorphism constraint means that the place to which the edges stick the IH is not relevant. The scalar constraint is nontrivial. However, since $(j, m)$ characterize the bulk almost at the $\mathrm{IH}$, it is assumed that the bulk scalar constraint does not affect $(j, m)$. At the IH, we do not consider the scalar constraint since the lapse function disappears. If we require that the horizon should be invariant under the diffeomorphism and the $\mathrm{U}(1)$ gauge transformation, the horizon area $A$ is fixed as

$$
A=4 \pi \gamma k
$$

where $k$ is a natural number and it is the level of the ChernSimons theory. In addition to this condition, it is required that we should fix an ordering $\left(j_{1}, j_{2}, \cdots, j_{n}\right)$. The area operator eigenvalue $A_{j}$ should satisfy

$$
\text { (i) } A_{j}=8 \pi \gamma \sum_{i} \sqrt{j_{i}\left(j_{i}+1\right)} \leq A \text {. }
$$


We mention other conditions. From the quantum GaussBonnet theorem, we require

$$
\text { (ii) } \sum_{i=1}^{n} b_{i}=0
$$

From the boundary condition between the IH and the bulk, we have

$$
\text { (iii) } b_{i}=-2 m_{i} \bmod k \text {. }
$$

All that we need to consider in number counting is (i), (ii), and (iii).

\section{NUMBER COUNTING}

Here, we consider number counting based on the ABCK framework. If we use (ii) and (iii), we obtain

$$
\text { (ii) } \sum_{i=1}^{n} m_{i}=n^{\prime} \frac{k}{2} \text {. }
$$

In [9], it was shown that this condition is irrelevant in number counting. Thus, we perform number counting only concentrating on (i) below.

For this purpose, there are two different points of view. The one adopted in the original paper $[1,8,9]$ counts the surface freedom $\left(b_{1}, b_{2}, \cdots, b_{n}\right)$. The second counts the freedom for both $j$ and $b[11,12]$.

We first consider the second possibility since (we suppose) it is easier to understand. To simplify the problem, we first consider the set $M_{k}$ by following [8], that is

$$
M_{k}:=\left\{\left(j_{1}, \cdots, j_{n}\right) \mid 0 \neq j_{i} \in \frac{Z}{2}, \sum_{i} j_{i} \leq \frac{k}{2}\right\} .
$$

Here, we also eliminate $A$ using (2.4). Let $N_{k}$ be the number of elements of $M_{k}$ plus 1 . Certainly,

$$
N(a) \leq N_{k},
$$

where $N(a)\left(a:=\frac{A}{8 \pi \gamma}\right)$ is the number of states which account for the entropy. Note that if $\left(j_{1}, \cdots, j_{n}\right) \in M_{k-1}$, then $\left(j_{1}, \cdots, j_{n}, \frac{1}{2}\right) \in M_{k}$. In the same way, for natural $0<$ $s \leq k$,

$$
\left(j_{1}, \cdots, j_{n}\right) \in M_{k-s} \Rightarrow\left(j_{1}, \cdots, j_{n}, \frac{s}{2}\right) \in M_{k} .
$$

Then, if we consider all $0<s \leq k$ and all the sequence $\left(j_{1}, \cdots, j_{n}\right) \in M_{k-s}$, we found that $\left(j_{1}, \cdots, j_{n}, \frac{s}{2}\right)$ form the entire set $M_{k}$. Moreover, for $s \neq s^{\prime}$,

$$
\left(j_{1}, \cdots, j_{n}, \frac{s}{2}\right) \neq\left(j_{1}, \cdots, j_{n}, \frac{s^{\prime}}{2}\right) \in M_{k} .
$$

The important point to remember is that we should include the condition $m_{i} \neq 0$ (or equivalently $b_{i} \neq 0$ ). Thus, each $j_{i}$ has freedom $2 j_{i}$ for the $j_{i}$ integer and the $2 j_{i}+1$ way for the $j_{i}$ half-integer. They are summarized as $2\left[\frac{2 j+1}{2}\right]$ where $[\cdots]$ is the integer parts. For this reason, the recursion relation is

$$
N_{k}=\sum_{s=1} 2[(s+1) / 2]\left(N_{k-s}-1\right)+1 .
$$

This is the point which has not been examined so far.

As a straight forward extension of this, we can consider $N(a)$, which is

$$
\begin{aligned}
N(a) & :=\left\{\left(j_{1}, \cdots, j_{n}\right) \mid 0 \neq j_{i} \in \frac{Z}{2}, \sum_{i} \sqrt{j_{i}\left(j_{i}+1\right)}\right. \\
& \left.\leq \frac{k}{2}=a\right\} .
\end{aligned}
$$

In this case, we obtain the recursion relation

$$
\begin{aligned}
N(a)= & 2 N(a-\sqrt{3} / 2)+2 N(a-\sqrt{2})+\cdots \\
& +2\left[\frac{2 j+1}{2}\right] N\left(a-\sqrt{j_{i}\left(j_{i}+1\right)}\right)+\cdots \\
& +\left[\sqrt{4 a^{2}+1}-1\right] .
\end{aligned}
$$

If we notice that the solution of $\sqrt{j_{i}\left(j_{i}+1\right)}=a$ is $j_{i}=$ $\left(\sqrt{4 a^{2}+1}-1\right) / 2$, the meaning of $\left[\sqrt{4 a^{2}+1}-1\right]$ is obvious.

If we use the relation

$$
N(a)=C e^{\left[\left(A \gamma_{M}\right) /(4 \gamma)\right]},
$$

where $C$ is a constant that was obtained in [9], we obtain

$$
1=\sum_{j=Z / 2} 2\left[\frac{2 j+1}{2}\right] \exp \left(-2 \pi \gamma_{M} \sqrt{j(j+1)}\right),
$$

by plugging (3.9) into (3.8) and taking the limit $A \rightarrow \infty$. Then if we require $S=A / 4$, we have $\gamma=\gamma_{M}$. This is the extension of $[11,12]$. In this case, $\gamma=0.26196 \cdots$.

Next, we consider the first possibility that counts only the surface freedom. This means that even if $\left(j_{1}, j_{2}, \cdots, j_{n}\right)$ is different, it is regarded as the same surface state if the horizon area and $\left(b_{1}, b_{2}, \cdots, b_{n}\right)$ are same. For example, $\left(j_{1}, j_{2}\right)=(3 / 2,1 / 2)$ and $(1 / 2,3 / 2)$ both give the possibility $\left(b_{1}, b_{2}\right)=(-1,-1)$. Then, it should not be distinguished in this description.

What should we do in this number counting? This following is the method taken in [8]; i.e., we rewrite (2.5) as

$$
(i)^{\prime} \quad 8 \pi \gamma \sum_{i} \sqrt{\left|m_{i}\right|\left(\left|m_{i}\right|+1\right)} \leq A
$$

Let us compare (3.4) with

$$
\left(m_{1}, \cdots, m_{n}\right) \in M_{k-s} \Rightarrow\left(m_{1}, \cdots, m_{n}, \pm \frac{s}{2}\right) \in M_{k}
$$

At first glance, it might seem that we abandon the freedom $m_{n+1}=-\frac{s}{2}+1, \cdots \frac{s}{2}-1$. However, this is not the case since we obtain that freedom from $M_{k-s+2}, M_{k-s+4}, \cdots$. It 
is the crucial difference from (3.4), where the freedom of $j$ is counted. In this way, we have the relation

$$
N_{k}=\sum_{s=1} 2\left(N_{k-s}-1\right)+1 .
$$

Therefore, we obtain (1.6).

\section{CONCLUSIONS AND DISCUSSION}

In this paper, we have considered two possibilities for the number of states of black holes in the ABCK framework. One of them gives a new value for the Immirzi parameter. From these results, we consider whether or not there is a consistency between the area spectrum in LQG and the area spectrum in the quasinormal mode. Since the area spectrum obtained from the quasinormal mode is $d A=4 \ln 3$, it is obvious that we do not have the same consistency if we adopt the Immirzi parameter determined by (1.6) or (3.10). Then, how about the case in which only $j=j_{\min }$ survives, as considered in [13]? Unfortunately, both (1.6) and (3.10) do not provide consistency that is different from the case in (1.7). This means that if we take the consistency to the quasinormal mode seriously, we will need new considerations.

Finally, we want to consider which of the two candidates is the better choice. The reason why only surface degree was counted in $[1,8,9]$ was to separate surface degree from the bulk freedom. If we admit $j$ as an independent variable, it is difficult to separate it from other bulk freedoms since that in the bulk can communicate with infinity. However, as pointed out in [12], it is $j$ that determines area eigenvalue, and other bulk variables are irrelevant. Moreover, since quantum horizons would fluctuate [27], it may be a problem to consider the $\mathrm{IH}$ as a sharp boundary. For these reasons, it is too early to abandon the possibility that we could count $j$ as an independent variable. Of course, it is also important to consider the other method in the calculating the number of freedom as in [28]. We also want to examine both of these possibilities in future.

\section{ACKNOWLEDGMENTS}

We would like to thank Professor Jerzy Lewandowski for useful comments and thank Professor Lee Smolin for hospitality when one of us stayed at the Perimeter Institute. This work was partially supported by The 21st Century COE Program (Holistic Research and Education Center for Physics Self-Organization Systems) at Waseda University. This work was supported in part by a JSPS Grant-in-Aid, No. 154568 (T. T.). This work was also supported in part by a Grant-in-Aid for the 21st Century COE "Center for Diversity and Universality in Physics."
[1] A. Ashtekar, J. Baez, A. Corichi, and K. Krasnov, Phys. Rev. Lett. 80, 904 (1998); A. Ashtekar, J. Baez, and K. Krasnov, Adv. Theor. Math. Phys. 4, 1 (2000).

[2] For review, see, e.g., M. Bojowald and H. A. MoralesTecotl, Lect. Notes Phys. 646, 421 (2004).

[3] M. Bojowald, Phys. Rev. Lett. 95, 061301 (2005).

[4] C. Rovelli and L. Smolin, Phys. Rev. D 52, 5743 (1995).

[5] C. Rovelli and L. Smolin, Nucl. Phys. B442, 593 (1995); B456, 753E (1995).

[6] A. Ashtekar and J. Lewandowski, Classical Quantum Gravity 14, A55 (1997).

[7] G. Immirzi, Nucl. Phys. Proc. Suppl. 57, 65 (1997).

[8] M. Domagala and J. Lewandowski, Classical Quantum Gravity 21, 5233 (2004).

[9] K.A. Meissner, Classical Quantum Gravity 21, 5245 (2004).

[10] A. Alekseev, A. P. Polychronakos, and M. Smedback, Phys. Lett. B 574, 296 (2003); A. P. Polychronakos, Phys. Rev. D 69, 044010 (2004).

[11] I. B. Khriplovich, gr-qc/0409031; gr-qc/0411109.

[12] A. Ghosh and P. Mitra, Phys. Lett. B 616, 114 (2005).

[13] S. Alexandrov, gr-qc/0408033; O. Dreyer, F. Markopoulou, and L. Smolin, hep-th/0409056.

[14] A. Strominger and C. Vafa, Phys. Lett. B 379, 99 (1996); J. M. Maldacena and A. Strominger, Phys. Rev. Lett. 77,
428 (1996).

[15] For review, see, e.g., J. Natario and R. Schiappa, hep-th/ 0411267.

[16] O. Dreyer, Phys. Rev. Lett. 90, 081301 (2003).

[17] S. Hod, Phys. Rev. Lett. 81, 4293 (1998).

[18] L. Motl, Adv. Theor. Math. Phys. 6, 1135 (2003); L. Motl and A. Neitzke, ibid. 7, 307 (2003).

[19] G. Kunstatter, Phys. Rev. Lett. 90, 161301 (2003).

[20] D. Birmingham, Phys. Lett. B 569, 199 (2003).

[21] T. Tamaki and H. Nomura, Phys. Rev. D 70, 044041 (2004).

[22] A. J.M. Medved, D. Martin, and M. Visser, Classical Quantum Gravity 21, 1393 (2004); 21, 2393 (2004).

[23] T. Padmanabhan, Classical Quantum Gravity 21, L1 (2004); T. R. Choudhury and T. Padmanabhan, Phys. Rev. D 69, 064033 (2004).

[24] J. Kettner, G. Kunstatter, and A. J. M. Medved, Classical Quantum Gravity 21, 5317 (2004).

[25] S. Das and S. Shankaranarayanan, Classical Quantum Gravity 22, L7 (2005).

[26] A. Ashtekar, A. Corichi, and K. Krasnov, Adv. Theor. Math. Phys. 3, 419 (1999).

[27] M. Bojowald and R. Swiderski, Phys. Rev. D 71, 081501 (2005).

[28] A. Dasgupta, J. Cosmol. Astropart. Phys. 08 (2003) 004. 